\title{
TRADUÇÃO E ADAPTAÇÃO TRANSCULTURAL PARA A LIINGUA PORTUGUESA DO "SWISS SPINAL STENOSIS QUESTIONNAIRE"
}

\author{
TRANSLATION AND CROSS-CULTURAL ADAPTATION TO PORTUGUESE \\ OF "SWISS SPINAL STENOSIS QUESTIONNAIRE"
}

\author{
TRADUCCIÓN TRANSCULTURAL Y ADAPTACIÓN AL PORTUGUÉS \\ DE "SWISS SPINAL STENOSIS QUESTIONNAIRE"
}

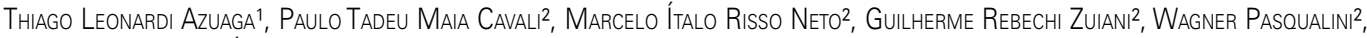 \\ IVAN GUIDOLIN VEIGA2 É ÉLCIO LANDIM³
}

\begin{abstract}
RESUMO
Objetivo: Traduzir e adaptar transculturalmente para a língua portuguesa o questionário Swiss Spinal Stenosis Questionnaire. Método: 1) tradução inicial; 2) retrotradução; 3) pré - teste; 4) teste definitivo. Resultados: Aplicamos a versão em português em 27 pacientes com estenose vertebral. Foram realizadas mudanças de termos e expressões que não foram entendidas pelos pacientes durante o pré-teste e realizada a versão final em consenso. A versão final do questionário foi aplicada com 100\% de entendimento pelos pacientes. Conclusão: Disponibiliza-se assim a versão final em português do Swiss Spinal Stenosis Questionnaire. A validação desta versão já está em desenvolvimento.
\end{abstract}

Descritores: Coluna vertebral; Estenose espinal; Tradução; Questionários.

\begin{abstract}
Objective: Translate and culturally adapt to Brazilian-Portuguese the "Swiss Spinal Stenosis Questionnaire". Method: 1) initial translation; 2) back-translation; 3) pre-test; 4) final test. Results: The Brazilian- Portuguese version was administered to 27 patients with spinal stenosis. Changes were made to terms and expressions that were not understood by patients during the pre-test, and the final version was drawn up in consensus. This final version was applied again, resulting in $100 \%$ of patients understanding. Conclusion: Therefore, the final version is available from the Swiss Spinal Stenosis Questionnaire in Brazilian-Portuguese. The validation of this version is already in progress.
\end{abstract}

Keywords: Spine, Spinal stenosis; Translation; Questionnaires.

RESUMEN

Objetivo: Traducir y adaptar culturalmente para el portugués de Brasil al "Swiss Spinal Stenosis Questionnaire". Método: 1) traducción inicial, 2) retrotraducción, 3) pre - test y 4) teste final. Resultados: La versión en portugués de Brasil fue administrada a 27 pacientes con estenosis espinal. Se hicieron cambios a los términos y expresiones que no fueron comprendidas por los pacientes durante el pre-test y la versión final se completó en consenso. El cuestionario final fue aplicado con un 100\% de comprensión de los pacientes. Conclusión: Por lo tanto, la versión final del cuestionario de la estenosis espinal suizo. La validación de esta versión está ya en progreso.

Descriptores: Columna vertebral; Estenosis Espinal; Traducctión; Cuestionarios.

\section{INTRODUÇÃO}

A estenose vertebral, definida como estreitamento do canal vertebral, recesso lateral ou dos forames neurais devido a hipertrofia de qualquer estrutura osteocartilaginosa e ligamentar que pode resultar em compressão neurológica ou vascular em um ou mais níveis, foi descrita por Verbiest em $1954^{1,2}$

A incidência de estenose lombar aumenta durante a quinta década de vida e varia de $1,7 \%$ a $8 \%^{3}$, sem predominância por sexo.

Estenose vertebral é um processo dinâmico e progressivo, pode ser melhor compreendida com as mudanças patológicas que ocorrem durante o envelhecimento. A compressão pode ocorrer em uma ou mais regiões anatômicas: central, recesso lateral, foraminal ou extra-foraminal. Como etiologia temos: congênita, adquirida ou mista ${ }^{4}$. A forma degenerativa é a mais comum, onde as alterações osteoartríticas, como diminuição da altura e protusão do disco intervertebral, espessamento do ligamento amarelo, formações osteofitárias, espondilolistese degenerativa e hipertrofia das articulações zigoapofisárias, resultam em diminuição da área do canal vertebral. Existem três formas de canal vertebral: redonda, oval e em trevo. A forma em trevo esta presente na maioria dos casos sintomáticos ${ }^{5}$.

Estenose lombar é uma causa comum de lombalgia e de dor nas pernas na população mais idosa ${ }^{6}$, existe uma dissociação entre a sintomatologia apresentada pelo paciente e os exames de imagem, ou seja, um paciente com uma estenose discreta pode apresentar uma dor importante, sendo necessário portanto o uso de questionários específicos para uma melhor avaliação quanto ao tipo de tratamento, conservador ou cirúrgico. Apesar da lombalgia ser um dos primeiros sintomas a aparecer, dor nas pernas é o sintoma

1. Médico Ortopedista eTraumatologista, Especializando em Cirurgia da Coluna Vertebral da Universidade Estadual de Campinas (Unicamp) - Campinas SP, Brasil. 2. Médico Assistente do Departamento de Ortopedia eTraumatologia da FCM (Unicamp) - Campinas, SP, Brasil.

3. Professor Doutor do Departamento de Ortopedia eTraumatologia da FCM (Unicamp) e Chefe do Grupo de Cirurgia de Coluna - Campinas, SP, Brasil.

Trabalho realizado no Ambulatório de Coluna do Departamento de Ortopedia eTraumatologia da Universidade Estadual de Campinas (Unicamp), Campinas, São Paulo, Brasil.

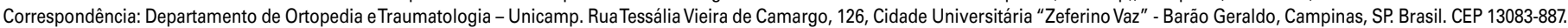
mrisso@me.com / thiazuaga@gmail.com 
mais comum, presente em $80 \%$ dos pacientes com esta patologia 6 .

Um grande número de questionários de qualidade de vida relacionados a saúde, foram desenvolvidos nos últimos anos, sendo instrumentos fundamentais nas análises das pesquisas científicas. Em 1996 Stucki et al. ${ }^{7}$ criaram um questionário específico para estenose vertebral, o Swiss Spinal Stenosis Questionnaire (SSS) que consiste em três subescalas: gravidade dos sintomas, função física e grau de satisfação com cirurgia. As questões referentes a gravidade dos sintomas, 1 a 7 do Anexo 1, são subdivididos em questões sobre dor (1 a 4) e sobre sintomas neuroisquêmicos (5 a 7), as questões de 8 a 12 da Anexo 1 são referentes à função física, e as questões de 13 a 18 da Anexo 1 são sobre a satisfação com procedimento cirúrgico.

Cada questão na subescala da gravidade dos sintomas (questões 1 a 7) deve receber de 1 a 5 pontos de acordo com a resposta dada e cada questão nas subescalas de função física (questões de 8 a 12) e de satisfação com a cirurgia (questões de 13 a 18) deve receber de 1 a 4 pontos também de acordo com a resposta apresentada. O resultado final do questionário é expresso como uma porcentagem do valor máximo possível e quanto maior o escore, pior o quadro clínico.

O Swiss Spinal Stenosis Questionnaire é um dos questionários mais reprodutíveis ${ }^{8}$ e considerado pela North American Spine Society (NASS) como o melhor e mais específico questionário para estenose lombar ${ }^{9}$. Para utilizar este questionário em outras culturas, os itens devem ser traduzidos e adaptados culturalmente para manter sua validade ${ }^{10,11}$. Esta adaptação cultural para uso em outro país, cultura e/ou idioma precisa de um método para a tradução e equivalência do questionário original ${ }^{11}$, para tanto utilizamos os métodos propostos por Guillemin et $\mathrm{al}^{12}$.

Este estudo tem por objetivo realizar a tradução e adaptação transcultural para a língua portuguesa com adaptação para a população brasileira do Swiss Spinal Stenosis Questionnaire, utilizado para estenose lombar.

\section{MATERIAIS E MÉTODOS}

O método de tradução e validação transcultural para a língua portuguesa do "Swiss Spinal Stenosis Questionnaire" consistiu dos critérios descritos por Guillemin et al, descrito a seguir.

\section{Tradução Inicial}

A versão original em inglês do "Swiss Spinal Stenosis Questionnaire" foi traduzido para a língua portuguesa por dois tradutores juramentados, independentes e qualificados. Formado um comitê com cinco ortopedistas, sendo quatro especialistas em cirurgia da coluna e um especializando em cirurgia da coluna. O comitê avaliou as duas traduções realizadas, analisando cada item do questionário, para observar possíveis distorções e sua aplicabilidade à realidade dos pacientes. Após essa análise, a versão número 1 em português foi obtida.

\section{Retrotradução}

Foi realizado a tradução para o idioma original, da versão número 1, por outros dois tradutores independentes, nativos da língua inglesa, com conhecido dos dois idiomas (português e inglês) e sem conhecimento do objetivo do estudo. Essas duas traduções foram avaliadas pelo comitê, para análise de discrepâncias e divergências entre as versões. Com isto chegamos a versão número dois em português.

\section{Pré-teste}

Aplicamos a versão número 2, que manteve as características conceituais do questionário original, a fim de verificar a equivalência da versão final do questionário e avaliar erros cometidos durante a tradução do mesmo.

Vinte e sete pacientes com diagnóstico de estenose do canal vertebral, sendo vinte e dois pré-operatório e cinco submetidos a procedimento cirúrgico, foram submetidos à aplicação da versão número 2, para avaliação da compreensão do questionário.

Como todas as questões e termos foram bem compreendidos, a versão número 2 em português tornou-se a versão final do questionário traduzido.

\section{Teste Definitivo}

Reaplicamos a versão final nos mesmos pacientes selecionados.

\section{Pacientes}

Participaram deste estudo vinte e sete pacientes com estenose do canal vertebral lombar acompanhados no ambulatório de Cirurgia da Coluna do Hospital das Clínicas da Universidade Estadual de Campinas (UNICAMP).

\section{Critérios de Inclusão}

Pacientes de 50 a 80 anos, independentemente de sexo e raça, apresentando sintomas de claudicação neurogênica uni ou bilateral (definida por dor nas nádegas ou membros inferiores causadas por ortostatismo ou deambulação e melhora com repouso ou com flexão do tronco) com diagnóstico de estenose lombar por tomografia computadorizada e/ou ressonância magnética.

\section{Aplicação do questionários}

O questionário foi aplicado nos pacientes pelo coordenador do trabalho, por entrevista simples. Os itens foram lidos pelo pesquisador para os pacientes e estes deveriam responder se compreendiam e comentar o que entenderam de cada item. Com isto obtivemos uma nova versão modificada e atualizada, tornando-se esta a versão brasileira do "Swiss Spinal Stenosis Questionnaire".

\section{RESULTADOS}

Chegamos a versão de consenso após encontro dos tradutores juramentados e pesquisadores. Durante a fase de retrotradução, tradutores e pesquisadores avaliaram e compararam as três versões (original, consensual e retrotraduções). Foram realizadas algumas alterações gramaticais para manter equivalência entre as palavras, idiomas e adaptação transcultural.

Como houve entendimento por todos os pacientes no que diz respeito à semântica da versão de consenso, esta tornou-se a versão final para língua portuguesa do Swiss Spinal Stenosis Questionnaire com adaptação para a população brasileira.

O tempo médio de aplicação do questionário foi de 7,5 minutos, variando entre cinco e dez minutos. A versão original, traduções, retrotraduções e versão de consenso se encontra no Anexo 1.

\section{DISCUSSÃO}

Devido o aumento da expectativa de vida, com o envelhecimento da população e com melhores métodos diagnósticos, houve um aumento no número de pacientes diagnosticados com estenose lombar.

Claudicação neurogênica é um dos primeiros sintomas, causando dor lombar baixa, nádegas e pernas, além de parestesia e fraqueza, o que dificulta para o paciente deambular. A quantidade de cirurgias para estenose vertebral tem aumentado nas últimas décadas e tende a aumentar com o envelhecimento a população ${ }^{13}$.

O tratamento de estenose lombar pode ser indicado e avaliado por questionários que levam em conta dor, satisfação e incapacida$\mathrm{de}^{8}$, como o Swiss Spinal Stenosis Questionnaire, SF-36, Oswestry Disability Index (ODI), Roland Morris, entre outros.

O SSS, já considerado pela NASS como o melhor e mais específico questionário para estenose lombar, tem vantagem em relação aos outros. O SF-36, um dos mais utilizados para avaliação da saúde em geral e qualidade de vida, apresenta 36 questões, divididas em componentes físico e mental, não avalia déficit neurológico como o SSS (questões 5 a 7). O ODI também é um dos instrumentos mais utilizados para avaliar função espinhal, avalia dor e atividade física, porém como o SF-36 não avalia déficit neurológico. 
O SSS tem a capacidade de avaliar essas variáveis que esses outros protocolos avaliam, com a vantagem de avaliar a parte neurológica, apresentar uma seção específica para avaliação pós-operatória, ser de fácil entendimento e fácil reprodutibilidade.

Estes questionários são realizados na língua inglesa e do nosso ponto de vista e de acordo com a literatura ${ }^{12}$ necessitam de adaptação para utilização em uma população de outra cultura, mantendo a equivalência semântica e não apenas literal durante o processo de tradução.
Para isso adotamos o método proposto por Guillemin et al. ${ }^{12}$, após a tradução transcultural, 100\% dos pacientes compreenderam os itens.

\section{CONCLUSÃO}

Com a elaboração da versão brasileira do Swiss Spinal Stenosis Questionnaire, temos um questionário específico para estenose lombar, traduzido e adaptado à cultura brasileira.

\section{REFERÊNCIAS}

1. Sirvanci M, Bhatia M, Ganiyusufoglu KA, Duran C, Tezer M, Ozturk C, et al. Degenerative lumbar spinal stenosis: correlation with Oswestry Disability Index and MR imaging. Eur Spine J. 2008;17(5):679-85.

2. Rodrigues LM, Ueno FH, Fujiki EN, Milani C. Estudo prospectivo comparativo entre pseudoartrose e fusão óssea na estenose de canal lombar. Acta Ortop Bras. 2011; 19(3):159-62.

3. De Villiers PD, Booysen EL. Fibrous spinal stenosis. A report on 850 myelograms with a water-soluble contrast medium. Clin Orthop Relat Res. 1976;(115):140-4.

4. Arnoldi CC, Brodsky AE, Cauchoix J, Crock HV, Dommisse GF, Edgar MA, et al. Lumbar spinal stenosis and nerve root entrapment syndromes. Definition and classification. Clin Orthop Relat Res. 1976;(115):4-5.

5. Bolender NF, Schönström NS, Spengler DM. Role of computed tomography and myelography in the diagnosis of central spinal stenosis. J Bone Joint Surg Am. 1985;67(2):240-6.

6. Arbit E, Pannullo S. Lumbar stenosis: a clinical review. Clin Orthop Relat Res. 2001;(384):137-43

7. Stucki G, Daltroy L, Liang MH, Lipson SJ, Fossel AH, Katz JN. Measurement properties of a self-administered outcome measure in lumbar spinal stenosis. Spine (Phila Pa 1976). $1996 ; 21(7): 796-803$

8. Pratt RK, Fairbank JC, Virr A. The reliability of the Shuttle Walking Test, the Swiss Spinal Stenosis Questionnaire, the Oxford Spinal Stenosis Score, and the Oswestry Disability Index in the assessment of patients with lumbar spinal stenosis. Spine (Phila Pa 1976). 2002:27(1):84-91.

9. North American Spine Society. Evidence Based Clinical Guidelines for Multidisciplinary Spine Care: diagnosis and treatment of degenerativelumbar spinal stenosis . Burr Ridge, IL : North American Spine Society ; 2007

10. Gonçalves CCK, Peccin MS, Almeida GJM, Cohen M. Tradução, validação e adaptação cultural da escala de atividade esportiva. Acta Ortop Bras. 2007;15:246-50.

11. Vilete L, Figueira I, Coutinho E. Adaptação transcultural para o português do Social Phobia Inventory (SPIN) para utilização entre estudantes adolescentes. Rev Psiquiatr Rio Gd Sul. 2006;28:40-8.

12. Guillemin F, Bombardier C, Beaton D. Cross-cultural adaptation of health-related quality of life measures: literature review and proposed guidelines. J Clin Epidemiol. 1993;46(12):1417-32

13. Tomkins CC, Battié MC, Hu R. Construct validity of the physical function scale of the Swiss Spinal Stenosis Questionnaire for the measurement of walking capacity. Spine (Phila Pa 1976). 2007;32(17):1896-901.

Anexo 1. Versões utilizadas do "Swiss Spinal Stenosis Questionnaire: original, tradução1, tradução 2, retrotradução 1, retrotradução 2 e versão final de consenso".

\begin{tabular}{|c|c|c|}
\hline Original & Tradução 1 & Tradução 2 \\
\hline $\begin{array}{l}\text { In the past month, how would you describe: } \\
\text { 1. The pain you have had on the average, including } \\
\text { pain in your back and buttocks, as well as pain that } \\
\text { goes down the legs? } \\
\text { ( ) None. } \\
\text { ( ) Mild. } \\
\text { ( ) Moderate. } \\
\text { ( ) Severe. } \\
\text { ( ) Very Severe. }\end{array}$ & $\begin{array}{l}\text { Como você descreveria no último mês: } \\
\text { 1. A dor que você teve, na média, incluindo dor nas } \\
\text { costas, nádegas ou dor irradiada para as pernas? } \\
\text { ( ) Nenhuma. } \\
\text { ( ) Leve. } \\
\text { ( ) Moderada. } \\
\text { ( ) Severa. } \\
\text { ( ) Muito Severa. }\end{array}$ & $\begin{array}{l}\text { Como você descreveria no último mês: } \\
\text { 1. A dor que sentiu em média, contando dor nas } \\
\text { costas e nádegas e a dor que desce pelas pernas? } \\
\text { ( ) Nenhuma. } \\
\text { ( ) Discreta. } \\
\text { ( ) Moderada. } \\
\text { ( ) Forte. } \\
\text { ( ) Muito Forte. }\end{array}$ \\
\hline $\begin{array}{l}\text { 2. How often have you had back, buttock, or leg pain? } \\
\text { ( ) Less than once a week. } \\
\text { ( ) At least once a week. } \\
\text { ( ) Every day, for at least a few minutes. } \\
\text { ( ) Every day for most of the day. } \\
\text { ( ) Every minute of the day. }\end{array}$ & $\begin{array}{l}\text { 2. Com qual frequência que você tem dor nas costas, } \\
\text { nádegas ou dor irradiada para as pernas? } \\
\text { ( ) Menos de uma vez por semana. } \\
\text { ( ) Pelo menos uma vez na semana. } \\
\text { ( ) Diariamente por alguns minutos. } \\
\text { ( ) Diariamente na maior parte do dia. } \\
\text { ( )Diariamente e durante o dia todo. }\end{array}$ & $\begin{array}{l}\text { 2. Com que frequência você teve dor nas } \\
\text { costas, nádegas ou nas pernas? } \\
\text { ( ) Menos de uma vez por semana. } \\
\text { ( ) Pelo menos uma vez por semana. } \\
\text { ( )Todos os dias, durante alguns minutos. } \\
\text { ( ) Todos os dias, a maior parte do tempo. } \\
\text { ( ) Todos os minutos do dia. }\end{array}$ \\
\hline $\begin{array}{l}\text { 3. The pain in your back or buttocks? } \\
\text { ( ) None. } \\
\text { ( ) Mild. } \\
\text { ( ) Moderate. } \\
\text { ( ) Severe. } \\
\text { ( ) Very Severe. }\end{array}$ & $\begin{array}{l}\text { 3. A dor nas costas ou nas nádegas? } \\
\text { ( ) Nenhuma. } \\
\text { ( ) Leve. } \\
\text { ( ) Moderada. } \\
\text { ( ) Severa. } \\
\text { ( ) Muito severa. }\end{array}$ & $\begin{array}{l}\text { 3. A dor nas costas ou nádegas? } \\
\text { ( ) Nenhuma. } \\
\text { ( ) Discreta. } \\
\text { ( ) Moderada. } \\
\text { ( ) Forte. } \\
\text { ( ) Muito Forte. }\end{array}$ \\
\hline $\begin{array}{l}\text { 4. The pain in your legs or feet? } \\
\text { ( ) None. } \\
\text { ( ) Mild. } \\
\text { ( ) Moderate. } \\
\text { ( ) Severe. } \\
\text { ( ) Very Severe. }\end{array}$ & $\begin{array}{l}\text { 4. A dor nas suas pernas ou pés? } \\
\text { ( ) Nenhuma. } \\
\text { ( ) Leve. } \\
\text { ( ) Moderada. } \\
\text { ( ) Severa. } \\
\text { ( ) Muito severa. }\end{array}$ & $\begin{array}{l}\text { 4. A dor nas pernas ou pés? } \\
\text { ( ) Nenhuma. } \\
\text { ( ) Discreta. } \\
\text { ( ) Moderada. } \\
\text { ( ) Forte. } \\
\text { ( ) Muito Forte. }\end{array}$ \\
\hline $\begin{array}{l}\text { 5. Numbness or tingling in your legs or feet? } \\
\text { ( ) None. } \\
\text { ( ) Mild. } \\
\text { ( ) Moderate. } \\
\text { ( ) Severe. } \\
\text { ( ) Very Severe. }\end{array}$ & $\begin{array}{l}\text { 5. Diminuição da sensibilidade ou formigamento nas } \\
\text { suas pernas ou pés? } \\
\text { ( ) Nenhuma. } \\
\text { ( ) Leve. } \\
\text { ( ) Moderada. } \\
\text { ( ) Severa. } \\
\text { ( ) Muito severa. }\end{array}$ & $\begin{array}{l}\text { 5. A dormência ou formigamento nas pernas e pés? } \\
\text { ( ) Nenhuma. } \\
\text { ( ) Discreta. } \\
\text { ( ) Moderada. } \\
\text { ( ) Forte. } \\
\text { ( ) Muito Forte. }\end{array}$ \\
\hline $\begin{array}{l}\text { 6. Weakness in your legs or feet? } \\
\text { ( ) None. } \\
\text { ( ) Mild. } \\
\text { ( ) Moderate. } \\
\text { ( ) Severe. } \\
\text { ( ) Very Severe. }\end{array}$ & $\begin{array}{l}\text { 6. Diminuição da força nas suas pernas ou pés? } \\
\text { ( ) Nenhuma. } \\
\text { ( ) Leve. } \\
\text { ( ) Moderada. } \\
\text { ( ) Severa. } \\
\text { ( ) Muito severa. }\end{array}$ & $\begin{array}{l}\text { 6. A fraqueza nas pernas ou pés? } \\
\text { ( ) Nenhuma. } \\
\text { ( ) Discreta. } \\
\text { ( ) Moderada. } \\
\text { ( ) Forte. } \\
\text { ( ) Muito Forte. }\end{array}$ \\
\hline
\end{tabular}


Anexo 1. Versões utilizadas do "Swiss Spinal Stenosis Questionnaire: original, tradução1, tradução 2, retrotradução 1, retrotradução 2 e versão final de consenso". 7. Problems with your balance?
( ) No, I've had no problems with balance.
( ) Yes, sometimes I feel my balance is off, or that I
am not surefooted.
( ) Yes, often I feel my balance is off, or that I am
not surefooted.
7. Alterações no seu equilíbrio?

( ) Não tenho problemas com equilíbrio.

( ) Sim, ás vezes perco o equilíbrio ou a firmeza nos pés.

( ) Sim, frequentemente perco o equilíbrio ou a firmeza nos pés.
In the past month, on a typical day:

8. How far have you been able to walk?

( ) More than 2 miles.

( ) More than 2 blocks, but less than 2 miles.

( ) More than 50 feet, but less than 2 miles.

( ) Less than 50 feet.

9. Have you taken walks outdoors or around the shops for pleasure?

( ) Yes, comfortably.

( )Yes, but sometimes with pain.

( ) Yes, but always with pain.

( ) No. No último mês, num dia típico:

8. Que distância você consegue percorrer andando?

( ) Mais de 3 quilômetros.

( ) Mais de 2 quarteirões mas menos que 3 quilômetros.

( ) Mais de 15 metros mas menos de 2 quateirões.

( ) Menos de 15 metros.

9. Você tem realizado caminhadas ao ar livre ou no shopping por diversão?

( ) Sim, confortavelmente.

( ) Sim, mas algumas vezes com dor.

( ) Sim, mas sempre com dor.

( ) Não.

10. Você tem saído para fazer compras na padaria/ mercado?

items?

( ) Yes, comfortably.

( ) Yes, but sometimes with pain.

( )Yes, but always with pain.

( ) No.

11. Have you walked around the different rooms in your house or apartment?

( ) Yes, comfortably.

( )Yes, but sometimes with pain.

( ) Yes, but always with pain.

( ) No.

12. Have you walked from your bedroom to the bathroom?

( ) Yes, comfortably.

( )Yes, but sometimes with pain

( )Yes, but always with pain.

( ) No.

If you have had treatment for your back or legs recently, how satisfied are you with

13. The overall result of your back operation?

( ) Very satisfied.

( ) Somewhat satisfied.

( ) Somewhat dissatisfied.

( ) Very dissatisfied.

14. Relief of pain after your operation?

( ) Very satisfied.

( ) Somewhat satisfied.

( ) Somewhat dissatisfied.

( ) Very dissatisfied.

15. The ability to walk after your operation?

( ) Very satisfied.

( ) Somewhat satisfied.

( ) Somewhat dissatisfied.

( ) Very dissatisfied.

16. Your ability to do housework, yardwork, or job after your operation?

( ) Very Satisfied.

( ) Somewhat satisfied.

( ) Somewhat dissatisfied.

( ) Very dissatisfied.

17. Your strength in your thighs, legs, and feet?

( ) Very satisfied.

( ) Somewhat satisfied.

( ) Somewhat dissatisfied.

( ) Very dissatisfied.

18. Your balance, or steadiness, on your feet?

( ) Very satisfied.

( ) Somewhat satisfied.

( ) Somewhat dissatisfied.

( ) Very dissatisfied.

( ) Muito satisfeito.

17. Sua força nas coxas, perna e pés?
( ) Sim, confortavelmente.

( ) Sim, mas algumas vezes com dor.

( ) Sim, mas sempre com dor.

( ) Não.

11. Você tem andado pelos diferentes cômodos de 11. Tem andado pela casa/apartamento?

sua casa/apartamento?

( ) Sim, confortavelmente.

( ) Sim, mas algumas vezes com dor.

( ) Sim, mas sempre com dor.

( ) Não.

12. Você anda do seu quarto até o banheiro?

( ) Sim, confortavelmente.

( ) Sim, mas algumas vezes com dor.

( ) Sim, mas sempre com dor.

( ) Não.

Se você foi submetido a tratamento para as suas costas/ pernas recentemente, quão satisfeito você está quanto a:

13. Resultado em geral da cirurgia da coluna

( ) Muito satisfeito.

( ) Parcialmente satisfeito.

( ) Parcialmente insatisfeito.

( ) Muito insatisfeito.

14. Melhora da dor após a cirurgia

( ) Muito satisfeito.

( ) Parcialmente satisfeito.

( ) Parcialmente insatisfeito.

( ) Muito insatisfeito.

15. Sua habilidade e m caminhar após a cirurgia?

( ) Parcialmente satisfeito.

( ) Parcialmente insatisfeito.

( ) Muito insatisfeito.

16. Sua capacidade de realizar tarefas domésticas, jardinagem ou seu trabalho após cirurgia?

( ) Muito satisfeito.

( ) Parcialmente satisfeito.

( ) Parcialmente insatisfeito.

() Muito insatisfeito.

( ) Muito satisfeito

( ) Parcialmente satisfeito.

( ) Parcialmente insatisfeito.

( ) Muito insatisfeito.

18. Seu equilíbrio ou firmeza nos seus pés?

( ) Muito satisfeito.

( ) Parcialmente satisfeito.

( ) Parcialmente insatisfeito.

( ) Muito insatisfeito.
7. A dificuldade de equilíbrio?

( ) Não, não tenho tido dificuldade de equilíbrio.

( ) Sim, às vezes fico sem equilíbrio ou sem firmeza nos pés.

( ) Sim, muitas vezes fico sem equilíbrio ou sem firmeza nos pés. No último mês, num dia típico:

8. Quanto você conseguiu andar?

( ) Mais de 3 quilômetros.

( ) Mais de 2 quarteirões, mas menos de 3 quilômetros.

( ) Mais de 15 metros, mas menos de 3 quilômetros. ( ) Menos de 15 metros.

9. você tem caminhado ao ar livre ou saído para ver lojas para se distrair?

( ) Sim, com conforto.

( ) Sim, mas às vezes com dor

( ) Sim, mas sempre com dor.

( ) Não.

10. Tem ido ao supermercado ou feito compras?

( ) Sim, com conforto.

( ) Sim, mas às vezes com dor

( ) Sim, mas sempre com dor.

( ) Não.

( ) Sim, com conforto.

( ) Sim, mas às vezes com dor

( ) Sim, mas sempre com dor.

( ) Não.

12. Tem andado do quarto pra o banheiro?

( ) Sim, com conforto.

( ) Sim, mas às vezes com dor.

( ) Sim, mas sempre com dor.

( ) Não.

Caso tenha recebido tratamento para as costas ou pernas recentemente, qual o seu grau de satisfacão com?

13. O resultado geral da operação das costas?

( ) Muito satisfeito (a)

( ) Mais ou menos satisfeito (a).

( ) Meio insatisfeito (a).

( ) Muito insatisfeito (a)

14. O alívio da dor após a operação?

( ) Muito satisfeito (a).

( ) Mais ou menos satisfeito (a).

() Meio insatisfeito (a).

( ) Muito insatisfeito (a)

15. A capacidade de caminhar depois da operação?

( ) Muito satisfeito (a).

( ) Mais ou menos satisfeito (a)

( ) Meio insatisfeito (a).

( ) Muito insatisfeito (a).

16. A capacidade de fazer serviços de casa, de jardinagem ou de trabalhar depois da operação?

( ) Muito satisfeito (a).

( ) Mais ou menos satisfeito (a).

( ) Meio insatisfeito (a).

( ) Muito insatisfeito (a).

17. A dor nas coxas, pernas e pés?

( ) Muito satisfeito (a).

( ) Mais ou menos satisfeito (a).

( ) Meio insatisfeito (a).

() Muito insatisfeito (a).

18. Seu equilíbrio ou estabilidade nos pés?

( ) Muito satisfeito (a).

( ) Mais ou menos satisfeito (a).

( ) Meio insatisfeito (a).

( ) Muito insatisfeito (a). 
Anexo 1. Versões utilizadas do "Swiss Spinal Stenosis Questionnaire: original, tradução1, tradução 2, retrotradução 1, retrotradução 2 e versão final de consenso".

\begin{tabular}{|c|c|c|}
\hline Retrotradução 1 & Retrotradução 2 & Versão de consenso \\
\hline How would you describe in the past month: & In the past month, how would you describe: & Como você descreveria no último mês: \\
\hline $\begin{array}{l}\text { 1) The pain you had in average, including pain in your } \\
\text { back, buttocks or radiating to your legs? } \\
\text { ( ) None. } \\
\text { ( ) Slight. } \\
\text { ( ) Moderate. } \\
\text { ( ) Severe. } \\
\text { ( ) Very severe. }\end{array}$ & $\begin{array}{l}\text { 1) The pain you have experienced on average, including } \\
\text { in your back, buttocks and that radiated to your legs? } \\
\text { ( ) None. } \\
\text { ( ) Light. } \\
\text { ( ) Moderate. } \\
\text { ( ) Severe. } \\
\text { ( ) Very severe. }\end{array}$ & $\begin{array}{l}\text { 1) A dor que sentiu em média, incluindo dor nas } \\
\text { costas, nádegas ou irradiada para as pernas? } \\
\text { ( ) Nenhuma. } \\
\text { ( ) Leve. } \\
\text { ( ) Moderada. } \\
\text { ( ) Forte. } \\
\text { ( ) Muito Forte. }\end{array}$ \\
\hline $\begin{array}{l}\text { 3) The pain in your back or buttocks? } \\
\text { ( ) None. } \\
\text { ( ) Slight. } \\
\text { ( ) Moderate. } \\
\text { ( ) Severe. } \\
\text { ( ) Very severe. }\end{array}$ & $\begin{array}{l}\text { 3) The pain in your back or buttocks? } \\
\text { ( ) None. } \\
\text { ( ) Light. } \\
\text { ( ) Moderate. } \\
\text { ( ) Severe. } \\
\text { ( ) Very severe. }\end{array}$ & $\begin{array}{l}\text { 3) A dor nas costas ou nádegas? } \\
\text { ( ) Nenhuma. } \\
\text { ( ) Leve. } \\
\text { ( ) Moderada. } \\
\text { ( ) Forte. } \\
\text { ( ) Muito Forte. }\end{array}$ \\
\hline $\begin{array}{l}\text { 4) The pain in your legs or feet? } \\
\text { ( ) None. } \\
\text { ( ) Slight. } \\
\text { ( ) Moderate. } \\
\text { ( ) Severe. } \\
\text { ( ) Very severe. }\end{array}$ & $\begin{array}{l}\text { 4) The pain in your legs or feet? } \\
\text { ( ) None. } \\
\text { ( ) Light. } \\
\text { ( ) Moderate. } \\
\text { ( ) Severe. } \\
\text { ( ) Very severe. }\end{array}$ & $\begin{array}{l}\text { 4) A dor nas suas pernas ou pés? } \\
\text { ( ) Nenhuma. } \\
\text { ( ) Leve. } \\
\text { ( ) Moderada. } \\
\text { ( ) Forte. } \\
\text { ( ) Muito Forte. }\end{array}$ \\
\hline $\begin{array}{l}\text { 5) Numbness or tingling in your legs or feet? } \\
\text { ( ) None. } \\
\text { ( ) Slight. } \\
\text { ( ) Moderate. } \\
\text { ( ) Severe. } \\
\text { ( ) Very severe. }\end{array}$ & $\begin{array}{l}\text { 5) Numbness or tingling in your legs or feet? } \\
\text { ( ) None. } \\
\text { ( ) Light. } \\
\text { ( ) Moderate. } \\
\text { ( ) Severe. } \\
\text { ( ) Very severe. }\end{array}$ & $\begin{array}{l}\text { 5) Dormência ou formigamento nas suas pernas ou pés? } \\
\text { ( ) Nenhuma. } \\
\text { ( ) Leve. } \\
\text { ( ) Moderada. } \\
\text { ( ) Forte. } \\
\text { ( ) Muito Forte. }\end{array}$ \\
\hline $\begin{array}{l}\text { 6) Decrease of strength in your legs or feet? } \\
\text { ( ) None. } \\
\text { ( ) Slight. } \\
\text { ( ) Moderate. } \\
\text { ( ) Severe. } \\
\text { ( ) Very severe. }\end{array}$ & $\begin{array}{l}\text { 6) Weakness in your legs or feet? } \\
\text { ( ) None. } \\
\text { ( ) Light. } \\
\text { ( ) Moderate. } \\
\text { ( ) Severe. } \\
\text { ( ) Very severe. }\end{array}$ & $\begin{array}{l}\text { 6) Diminuição da força nas suas pernas ou pés? } \\
\text { ( ) Nenhuma. } \\
\text { ( ) Leve. } \\
\text { ( ) Moderada. } \\
\text { ( ) Forte. } \\
\text { ( ) Muito Forte. }\end{array}$ \\
\hline On a normal day in the past month: & In the past month, o & No último mês, er \\
\hline $\begin{array}{l}\text { 8) How much can you walk? } \\
\text { ( ) More than } 3 \text { kilometers. } \\
\text { ( ) More than } 2 \text { blocks, but less than } 3 \text { kilometers. } \\
\text { ( ) More than } 15 \text { meters, but less than } 3 \text { kilometers. } \\
\text { () Less than } 15 \text { meters. }\end{array}$ & $\begin{array}{l}\text { 8) How far have you been able to walk? } \\
\text { ( ) More than } 3 \text { kilometers ( } 1.86 \text { mile). } \\
\text { ( ) More than two blocks, but less than } 3 \text { kilometers } \\
\text { ( } 1.86 \text { mile). } \\
\text { ( ) More than } 15 \text { meters ( } 49.21 \text { feet), but less than } 3 \\
\text { kilometers ( } 1.86 \text { mile). } \\
\text { ( ) Less than } 15 \text { meters ( } 49.21 \text { feet). }\end{array}$ & $\begin{array}{l}\text { 8) Quanto você consegue andar? } \\
\text { ( ) Mais de } 3 \text { Kilometros. } \\
\text { ( ) Mais de } 2 \text { quarteirões, mas menos de } 3 \text { Kilo- } \\
\text { metros. } \\
\text { ( ) Mais de } 15 \text { metros, mas menos de } 3 \text { kilometros. } \\
\text { ( ) Menos de } 15 \text { metros. }\end{array}$ \\
\hline $\begin{array}{l}\text { 9) Have you taken walks outdoors or gone out for fun? } \\
\text { ( Yes, comfortably. } \\
\text { ( Yes, but sometimes I am in pain. } \\
\text { ( Yes, but I am always in pain. } \\
\text { ( ) No. }\end{array}$ & $\begin{array}{l}\text { 9) Have you taken walks outdoors or gone out for } \\
\text { pleasure? } \\
\text { ( ) Yes, comfortably. } \\
\text { ( )Yes, but sometimes with pain. } \\
\text { ( )Yes, but always with pain. } \\
\text { ( ) No. }\end{array}$ & $\begin{array}{l}\text { 9) Você tem realizado caminhadas ao ar livre ou saí- } \\
\text { do para se distrair? } \\
\text { ( ) Sim, confortavelmente. } \\
\text { ( ) Sim, mas algumas vezes com dor. } \\
\text { ( ) Sim, mas sempre com dor. } \\
\text { ( ) Não. }\end{array}$ \\
\hline $\begin{array}{l}\text { 10) Have you been to the supermarket or shopping } \\
\text { ( )Yes, comfortably. } \\
\text { ( )Yes, but sometimes I am in pain. } \\
\text { ( )Yes, but I am always in pain. } \\
\text { ( ) No. }\end{array}$ & $\begin{array}{l}\text { 10) Have you gone shopping for groceries or other items? } \\
\text { ( ) Yes, comfortably. } \\
\text { ( )Yes, but sometimes with pain. } \\
\text { ( )Yes, but always with pain. } \\
\text { ( ) No. }\end{array}$ & $\begin{array}{l}\text { 10) Você tem ido ao supermercado ou feito compras? } \\
\text { ( ) Sim, confortavelmente. } \\
\text { ( ) Sim, mas algumas vezes com dor. } \\
\text { ( ) Sim, mas sempre com dor. } \\
\text { ( ) Não. }\end{array}$ \\
\hline $\begin{array}{l}\text { 11) Have you walked at your home/apartment? } \\
\text { ( Yes, comfortably. } \\
\text { ( YYes, but sometimes I am in pain. } \\
\text { ( )Yes, but I am always in pain. } \\
\text { ( ) No. }\end{array}$ & $\begin{array}{l}\text { 11) Have you walked around your house or apartment? } \\
\text { ( ) Yes, comfortably. } \\
\text { ( )Yes, but sometimes with pain. } \\
\text { ( )Yes, but always with pain. } \\
\text { ( ) No. }\end{array}$ & $\begin{array}{l}\text { 11) Você tem andado pela sua casa/apartamento? } \\
\text { ( ) Sim, confortavelmente. } \\
\text { ( ) Sim, mas algumas vezes com dor. } \\
\text { ( ) Sim, mas sempre com dor. } \\
\text { ( ) Não. }\end{array}$ \\
\hline
\end{tabular}


Anexo 1. Versões utilizadas do "Swiss Spinal Stenosis Questionnaire: original, tradução1, tradução 2, retrotradução 1, retrotradução 2 e versão final de consenso".

\begin{tabular}{|c|c|c|}
\hline $\begin{array}{l}\text { 12) Do you walk from your bedroom to the bathroom? } \\
\text { ( YYes, comfortably. } \\
\text { ( ) Yes, but sometimes I am in pain. } \\
\text { ( ) Yes, but I am always in pain. } \\
\text { ( ) No. }\end{array}$ & $\begin{array}{l}\text { 12) Have you walked from your bedroom to the bathroom? } \\
(\text { ) Yes, comfortably. } \\
\text { ( )Yes, but sometimes with pain. } \\
\text { ( )Yes, but always with pain. } \\
\text { ( ) No. }\end{array}$ & $\begin{array}{l}\text { 12) Você anda do seu quarto até o banheiro? } \\
\text { ( ) Sim, confortavelmente. } \\
\text { ( ) Sim, mas algumas vezes com dor. } \\
\text { ( ) Sim, mas sempre com dor. } \\
\text { ( ) Não. }\end{array}$ \\
\hline $\begin{array}{l}\text { If you have undergone treatment for your back/legs } \\
\text { recently, which is your level of satisfaction with: }\end{array}$ & $\begin{array}{l}\text { If you have undergone treatment for your back or } \\
\text { legs, how satisfied are you with: }\end{array}$ & $\begin{array}{l}\text { Se você foi submetido a tratamento para suas costas/ } \\
\text { pernas recentemente, qual seu grau de satisfação com: }\end{array}$ \\
\hline $\begin{array}{l}\text { 13) The general outcome of the spine surgery? } \\
\text { ( ) Very satisfied. } \\
\text { ( ) Somewhat satisfied. } \\
\text { ( ) Somewhat dissatisfied. } \\
\text { ( ) Very dissatisfied. }\end{array}$ & $\begin{array}{l}\text { 13) The overall result of your back surgery? } \\
\text { ( ) Very satisfied. } \\
\text { ( ) Somewhat satisfied. } \\
\text { ( ) Somewhat dissatisfied. } \\
\text { ( ) Very dissatisfied. }\end{array}$ & $\begin{array}{l}\text { 13) Resultado em geral da cirurgia da coluna? } \\
\text { ( ) Muito satisfeito (a). } \\
\text { ( ) Mais ou menos satisfeito (a). } \\
\text { ( ) Meio insatisfeito (a). } \\
\text { ( ) Muito insatisfeito (a). }\end{array}$ \\
\hline $\begin{array}{l}\text { 14) The improvement of the pain after the surgery? } \\
\text { ( ) Very satisfied. } \\
\text { ( ) Somewhat satisfied. } \\
\text { ( ) Somewhat dissatisfied. } \\
\text { ( ) Very dissatisfied. }\end{array}$ & $\begin{array}{l}\text { 14) Improvement in pain after surgery? } \\
\text { ( ) Very satisfied. } \\
\text { ( ) Somewhat satisfied. } \\
\text { ( ) Somewhat dissatisfied. } \\
\text { ( ) Very dissatisfied. }\end{array}$ & $\begin{array}{l}\text { 14) Melhora da dor após a cirurgia? } \\
\text { ( ) Muito satisfeito (a). } \\
\text { ( ) Mais ou menos satisfeito (a). } \\
\text { ( ) Meio insatisfeito (a). } \\
\text { ( ) Muito insatisfeito (a). }\end{array}$ \\
\hline $\begin{array}{l}\text { 16) Your ability to do household chores, gardening or } \\
\text { your work after the surgery? } \\
\text { ( ) Very satisfied. } \\
\text { ( ) Somewhat satisfied. } \\
\text { ( ) Somewhat dissatisfied. } \\
\text { ( ) Very dissatisfied. }\end{array}$ & $\begin{array}{l}\text { 16) Your ability to do work around the house or in the } \\
\text { garden or to do your job after surgery? } \\
(\text { ) Very satisfied. } \\
\text { ( ) Somewhat satisfied. } \\
\text { ( ) Somewhat dissatisfied. } \\
\text { ( ) Very dissatisfied. }\end{array}$ & $\begin{array}{l}\text { 16) Sua capacidade de realizar serviços de casa, jar- } \\
\text { dinagem ou seu trabalho após a cirurgia } \\
\text { ( ) Muito satisfeito (a). } \\
\text { ( ) Mais ou menos satisfeito (a). } \\
\text { ( ) Meio insatisfeito (a). } \\
\text { ( ) Muito insatisfeito (a). }\end{array}$ \\
\hline $\begin{array}{l}\text { 17) Your strength on your thighs, legs and feet? } \\
\text { ( ) Very satisfied. } \\
\text { ( ) Somewhat satisfied. } \\
\text { ( ) Somewhat dissatisfied. } \\
\text { ( ) Very dissatisfied. }\end{array}$ & $\begin{array}{l}\text { 17) The strength in your thighs, legs and feet? } \\
\text { ( ) Very satisfied. } \\
\text { ( ) Somewhat satisfied. } \\
\text { ( ) Somewhat dissatisfied. } \\
\text { ( ) Very dissatisfied. }\end{array}$ & $\begin{array}{l}\text { 17) Sua força nas coxas, pernas e pés? } \\
\text { ( ) Muito satisfeito (a). } \\
\text { ( ) Mais ou menos satisfeito (a). } \\
\text { ( ) Meio insatisfeito (a). } \\
\text { ( ) Muito insatisfeito (a). }\end{array}$ \\
\hline
\end{tabular}

